\title{
Relationship of Hemoglobin A1c with $\beta$ Cell Function and Insulin Resistance in Newly Diagnosed and Drug Naive Type 2 Diabetes Patients
}

\author{
Xinguo Hou, ${ }^{1}$ Jinbo Liu, ${ }^{1}$ Jun Song, ${ }^{1}$ Chuan Wang, ${ }^{1}$ Kai Liang, ${ }^{1}$ Yu Sun, ${ }^{1}$ Zeqiang Ma, ${ }^{2}$ \\ Weifang Yang, ${ }^{3}$ Chengqiao Li, ${ }^{4}$ Xiuping Zhang, ${ }^{5}$ Peng Lin, ${ }^{1}$ Lei Gong, ${ }^{1}$ Meijian Wang, \\ Fuqiang Liu, ${ }^{1}$ Wenjuan Li, ${ }^{1}$ Fei Yan, ${ }^{1}$ Jun Qin, ${ }^{1}$ Lingshu Wang, ${ }^{1}$ Jidong Liu, ${ }^{1}$ Ruxing Zhao, \\ Shihong Chen, ${ }^{6}$ and Li Chen ${ }^{1}$
}

${ }^{1}$ Department of Endocrinology, Qilu Hospital, Shandong University, Jinan, Shandong 250012, China
${ }^{2}$ China National Heavy Duty Truck Group Corporation Hospital, Jinan, Shandong 250116, China
${ }^{3}$ Lukang Hospital of Jining, Jining, Shandong 272141, China
${ }^{4}$ Department of Endocrinology, Second People's Hospital of Jining, Jining, Shandong 272049, China
${ }^{5}$ Shantui Community Health Center, Jining, Shandong 272000, China
${ }^{6}$ Department of Endocrinology, The Second Hospital of Shandong University, Jinan, Shandong 250012, China

Correspondence should be addressed to Shihong Chen; chenshihong26@163.com and Li Chen; chenli3@medmail.com.cn

Received 13 February 2015; Accepted 26 March 2015

Academic Editor: Francis M. Finucane

Copyright (C) 2016 Xinguo Hou et al. This is an open access article distributed under the Creative Commons Attribution License, which permits unrestricted use, distribution, and reproduction in any medium, provided the original work is properly cited.

Objective. To investigate changes in the glycated hemoglobin Alc (Alc) level and those in $\beta$ cell function and insulin resistance in newly diagnosed and drug naive type 2 diabetes patients and to evaluate the relationship between them. Design and Methods. A total of 818 newly diagnosed diabetic individuals who were $\geq 40$ years of age were recruited. The subjects were grouped by Alc values $(<6.5 \%, 6.5-7 \%, 7-8 \%, 8-9 \%$, and $\geq 9 \%)$. The homeostasis model assessment (HOMA) was used to evaluate pancreatic $\beta$ cell function (HOMA- $\beta$ ) and insulin resistance (HOMA-IR). ANOVA, $t$-tests, and binary logistic regression analysis were used for data analysis. Results. Compared with subjects with Alc values $<6.5 \%$, individuals with an Alc of $6.5-7 \%$ exhibited an increased HOMA- $\beta$ index. However, the HOMA- $\beta$ index was significantly decreased at Alc values $\geq 7 \%$ and further decreased by $9.3 \%$ and by $23.7 \%$, respectively, at Alc values of $7-8 \%$ and $8-9 \%$. As Alc increased to $\geq 9 \%$, a $62 \%$ reduction in $\beta$ cell function was observed, independently of age, gender, body mass index (BMI), blood pressure (BP), blood lipids, and hepatic enzyme levels. Meanwhile, insulin resistance was significantly increased with an increase in Alc values. Conclusions. Elevated Alc values $(\geq 7 \%)$ were associated with substantial reductions in $\beta$ cell function.

\section{Introduction}

The main pathophysiological defects responsible for type 2 diabetes mellitus (T2DM) include $\beta$ cell dysfunction and decreased insulin sensitivity [1]. In the presence of insulin resistance, progressive loss of $\beta$ cell function is a crucial defect [2]. Many factors including hyperglycemia and elevated free fatty acid accelerate $\beta$ cell deterioration [3]. Accumulating evidence has shown that sustained hyperglycemia is deleterious to $\beta$ cell function. The hemoglobin Alc (Alc) value is an integrated measure of mean glycemia over the preceding 8-12 weeks and is considered the "gold standard" for monitoring metabolic control in subjects with diabetes [4]. It has been reported that an increase in the Alc level is usually accompanied by a decline in pancreatic $\beta$ cell function. However, little is known about the relationship between the Alc level and $\beta$ cell function, especially in newly diagnosed and drug naive type 2 diabetic patients. This study was performed to investigate the changes in Alc along with $\beta$ cell function and insulin resistance in newly diagnosed and drug naive type 2 diabetic patients and to evaluate the relationship between them. 


\section{Materials and Methods}

2.1. Ethics Statement. The present work consists of one part of the baseline survey from the Risk Evaluation of cAncers in Chinese diabeTic Individuals: a lONgitudinal (REACTION) study, which was conducted among 259,657 adults, aged 40 years and older, in 25 communities across mainland China from 2011 to 2012 [5-8]. This study was approved by the Ruijin Hospital Ethics Committee of the Shanghai Jiao Tong University School of Medicine. Written informed consent was obtained from the study participants.

2.2. Study Population. A total of 10,028 subjects were recruited (40 years of age and older) in Shandong province from January to April 2012. Based on previous medical histories and OGTT, we selected 818 newly diagnosed and drug naive type 2 diabetes patients. The exclusion criteria consisted of (1) previously diagnosed hepatic disease, including fatty liver, liver cirrhosis, and autoimmune hepatitis; (2) previously diagnosed diabetes; and (3) any malignant disease. A total of 818 subjects (508 women) were eligible for the analysis.

2.3. Data Collection. The demographic characteristics, lifestyle, and previous medical histories were obtained by trained investigators through a standard questionnaire. All subjects underwent a baseline evaluation including body mass index (BMI), waist circumference (WC), and blood pressure (BP). Laboratory evaluations of fasting blood glucose (FBG), fasting insulin, cholesterol, triglyceride, ALT, and AST levels were also performed. OGTTs were conducted in all patients, using a glucose load containing the equivalent of $75 \mathrm{~g}$ of anhydrous glucose dissolved in water. The Alc level was measured by high-performance liquid chromatography (VARIANT II and D-10 Systems, BIO-RAD, USA). The homeostasis model assessment of insulin resistance (HOMA-IR) index was calculated as follows: fasting insulin concentration $(\mathrm{mIU} / \mathrm{L}) \times$ FBG concentration $(\mathrm{mmol} / \mathrm{L}) / 22.5$. The HOMA- $\beta$ index was calculated as follows: $20 *$ fasting insulin concentration $(\mathrm{mIU} / \mathrm{L}) /(\mathrm{FBG}$ concentration $(\mathrm{mmol} / \mathrm{L})-3.5)[9]$.

2.4. Definition. Diabetic patients who were diagnosed based on the 1999 World Health Organization (WHO) criteria $(\mathrm{FBG} \geq 126 \mathrm{mg} / \mathrm{dL}(7.0 \mathrm{mmol} / \mathrm{L})$ and/or $2 \mathrm{hPG} \geq 200 \mathrm{mg} / \mathrm{dL}$ $(11.1 \mathrm{mmol} / \mathrm{L}))$ [10] were identified after OGTTs. To explore the association between Alc and insulin resistance/ $\beta$ cell function, we divided the subjects into the following five groups according to the Alc values: $<6.5 \%, 6.5-7 \%, 7-8 \%, 8$ $9 \%$, and $\geq 9 \%$.

2.5. Statistical Analysis. Continuous variables with a normal distribution are expressed as the means \pm standard deviation (SD), and variables with a nonnormal distribution are presented as medians (interquartile range). Categorical variables are presented as numbers (\%). Between-group differences were evaluated with ANOVA. Binary logistic regression analysis was used to estimate the association between Alc levels and $\beta$ cell function/insulin resistance in three models. The following three models were constructed: Model $1=$ not adjusted; Model 2 = adjusted for age, gender, BMI, and WC; Model 3 = Model 2 plus systolic BP, diastolic BP, cholesterol, triglycerides, ALT, and AST values. A value of $P<0.05$ was considered statistically significant. The data were analyzed using the SPSS 16.0 software (SPSS, Inc., Chicago, IL, USA).

\section{Results}

3.1. Characteristics of Study Participants Grouped by Alc Category. We recruited a total of 818 newly diagnosed and drug naive diabetic subjects, including 508 females and 310 males with an average age of $60.4 \pm 9.6$ years. The subjects were divided into five groups according to their Alc levels. As shown in Table 1, no difference in gender, systolic BP, cholesterol, LDL-C, and AST levels were observed between groups. Individuals with an Alc of $7-8 \%$ were more likely to be older and stronger and have a higher diastolic BP than those with an $\mathrm{Alc}<6.5 \%$.

3.2. $\beta$ Cell Function and Insulin Resistance Changes in Different Alc Groups. We used the HOMA- $\beta$ index to assess $\beta$ cell function. As shown in Figure 1(a), compared to subjects with an Alc $<6.5 \%$, individuals with an Alc of 6.5-7\% exhibited increased $\beta$ cell function. By contrast, the HOMA- $\beta$ index was significantly decreased in individuals with an Alc $\geq 7 \%$. As Alc increased to $\geq 9 \%$, a $62 \%$ reduction in $\beta$ cell function was observed. We further compared $\beta$ cell function at different Alc levels in male and female subjects. Impaired $\beta$ cell function was observed in subjects with an Alc $\geq 8 \%$ in both male and female patients; the values were decreased by $26 \%$ and $48 \%$, respectively. Furthermore, the HOMA- $\beta$ index values in female patients were significantly higher than those in male patients with an Alc $\geq 9 \%$.

We further used the HOMA-IR index to assess insulin resistance in different Alc groups (Figure 1(b)). Insulin resistance increased significantly with increasing Alc levels. Compared with the Alc $<6.5 \%$ group, insulin resistance increased by $9 \%, 14 \%, 18 \%$, and $29 \%$ in individuals with Alc values of $6.5-7 \%, 7-8 \%, 8-9 \%$, and $\geq 9 \%$, respectively. In male patients, insulin resistance was significantly higher in individuals with an Alc $\geq 7 \%$ than in individuals with an Alc $<6.5 \%$, while in female patients, the HOMA-IR index was increased only in individuals with an Alc $\geq 9 \%$. No significant difference was observed between male and female patients.

3.3. Binary Logistic Regression Analysis. As shown in Table 3, we analyzed the association between increased Alc levels and impaired $\beta$ cell function using three models. We found that patients with Alc values of $8 \%-9 \%$ and $\geq 9 \%$ had a significantly decreased $\beta$ cell function (odds ratio $(\mathrm{OR})=2.45$ and 15.36, resp.). After adjusting for age, gender, BMI, and WC, these two groups still presented increased ORs (3.69 and 22.08, resp.). After further adjusting for systolic BP, diastolic $\mathrm{BP}$, cholesterol, triglycerides, ALT, and AST, the patients with A1c levels of $8 \%-9 \%$ and $\geq 9 \%$ also showed an increased risk of impaired $\beta$ cell function ( $\mathrm{OR}=4.19$ and 28.51 , resp.). 
TABLE 1: Characteristic of study participants grouped by Alc category.

\begin{tabular}{lcccccc}
\hline Characteristics & Group 1 & Group 2 & Group 3 & Group 4 & Group 5 & Total \\
\hline$N$ & 311 & 156 & 172 & 67 & 112 & 818 \\
Female (\%) & $198(63.7 \%)$ & $103(66.0 \%)$ & $109(63.4 \%)$ & $38(56.7 \%)$ & $60(53.6 \%)$ & $508(62.1 \%)$ \\
Age (years) & $59.44 \pm 9.91$ & $61.7 \pm 9.69$ & $61.56 \pm 9.23^{*}$ & $61.76 \pm 8.72$ & $58.55 \pm 8.77$ & $60.39 \pm 9.54$ \\
BMI (kg/m $\left.{ }^{2}\right)$ & $26.44 \pm 3.51$ & $27.72 \pm 3.19^{* *}$ & $27.99 \pm 3.31^{* * *}$ & $27.56 \pm 3.38$ & $26.36 \pm 2.86$ & $27.09 \pm 3.39$ \\
Wc (cm) & $87.7 \pm 10.16$ & $91.53 \pm 8.88^{* * *}$ & $91.51 \pm 10.25^{* * *}$ & $91.63 \pm 10.84^{* *}$ & $89.56 \pm 8.71$ & $89.81 \pm 09.95$ \\
Systolic BP (mmHg) & $146.15 \pm 20.63$ & $146.56 \pm 19.42$ & $145.76 \pm 19.85$ & $146.31 \pm 18.83$ & $147.99 \pm 21.11$ & $146.4 \pm 20.13$ \\
Diastolic BP (mmHg) & $83.96 \pm 12.49$ & $82.4 \pm 11.76$ & $81.02 \pm 11.34^{* *}$ & $80.31 \pm 10.69^{*}$ & $84.22 \pm 12.38$ & $82.78 \pm 12.02$ \\
FBG (mmol/L) & $7.47 \pm 1.58$ & $7.4 \pm 0.91$ & $7.96 \pm 1.14^{* *}$ & $8.86 \pm 1.73^{* * *}$ & $12.54 \pm 3.38^{* * *}$ & $8.37 \pm 2.47$ \\
Fasting insulin (uU/mL) & $9.7(7-13.95)$ & $11.3(7.8-15.8)$ & $9.7(6.93-14.6)^{*}$ & $9.9(7-14.8)$ & $7.4(5.23-11.1)^{* *}$ & $9.85(6.9-14)$ \\
Alc (\%) & $5.96 \pm 0.34$ & $6.7 \pm 0.14^{* * *}$ & $7.39 \pm 0.3^{* * *}$ & $8.33 \pm 0.28^{* * *}$ & $10.86 \pm 1.75^{* * *}$ & $7.27 \pm 1.75$ \\
Cholesterol (mmol/L) & $5.51 \pm 1.14$ & $5.68 \pm 1.01$ & $5.6 \pm 0.97$ & $5.58 \pm 0.95$ & $5.76 \pm 1.42$ & $5.6 \pm 1.11$ \\
Triglycerides (mmol/L) & $1.42(1.01-2.1)$ & $1.61(1.19-2.27)$ & $1.73(1.26-2.47)$ & $1.91(1.3-2.8)^{*}$ & $1.81(1.28-2.36)^{* * *}$ & $1.59(1.12-2.27)$ \\
LDL-C (mmol/L) & $3.3 \pm 0.89$ & $3.45 \pm 0.84$ & $3.4 \pm 0.83$ & $3.32 \pm 0.83$ & $3.64 \pm 0.95$ & $3.4 \pm 0.88$ \\
HDL-C (mmol/L) & $1.55 \pm 0.39$ & $1.44 \pm 0.27^{* *}$ & $1.36 \pm 0.28^{* * *}$ & $1.34 \pm 0.24^{* * *}$ & $1.48 \pm 0.46$ & $1.46 \pm 0.36$ \\
ALT (U/L) & $13.67 \pm 9.75$ & $15.58 \pm 11.06^{* *}$ & $16.94 \pm 13.29$ & $16.19 \pm 9.89$ & $15.71 \pm 10.08$ & $15.21 \pm 10.94$ \\
AST (U/L) & $20.74 \pm 9.32$ & $21.13 \pm 9.03$ & $22.434 \pm 9.67$ & $21.64 \pm 11.32$ & $19.27 \pm 8.97$ & $21.03 \pm 9.49$ \\
\hline
\end{tabular}

Data are mean \pm SD or median (interquartile range) or number (\%). BMI, body mass index; WC, waist circumference; BP, blood pressure; FBG, fasting blood glucose; LDL-C, low-density lipoprotein cholesterol; HDL-C, high-density lipoprotein cholesterol; ALT, alanine aminotransferase; AST, aspartate aminotransferase. ${ }^{*} P<0.05$ compared with Group $1 ;{ }^{* *} P<0.01$ compared with Group $1 ;{ }^{* *} P<0.01$ compared with Group 1.

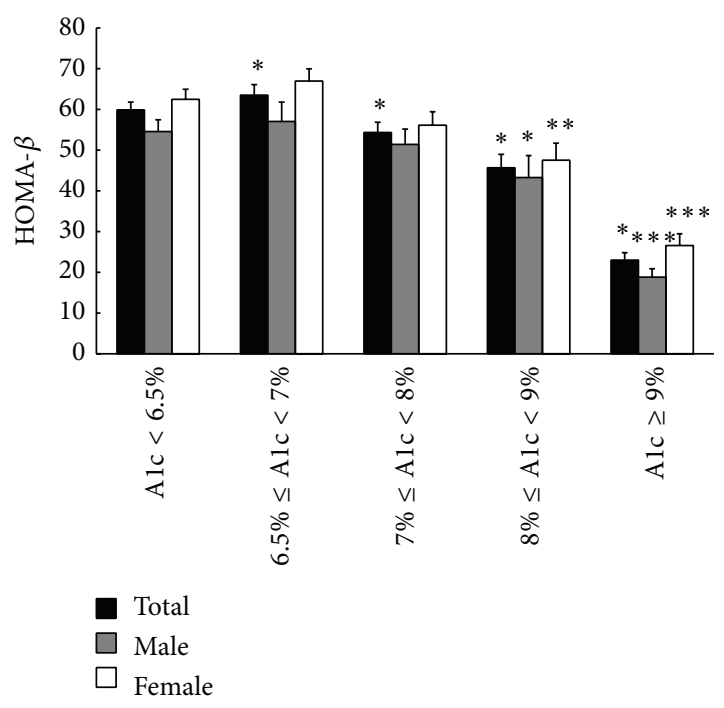

(a)

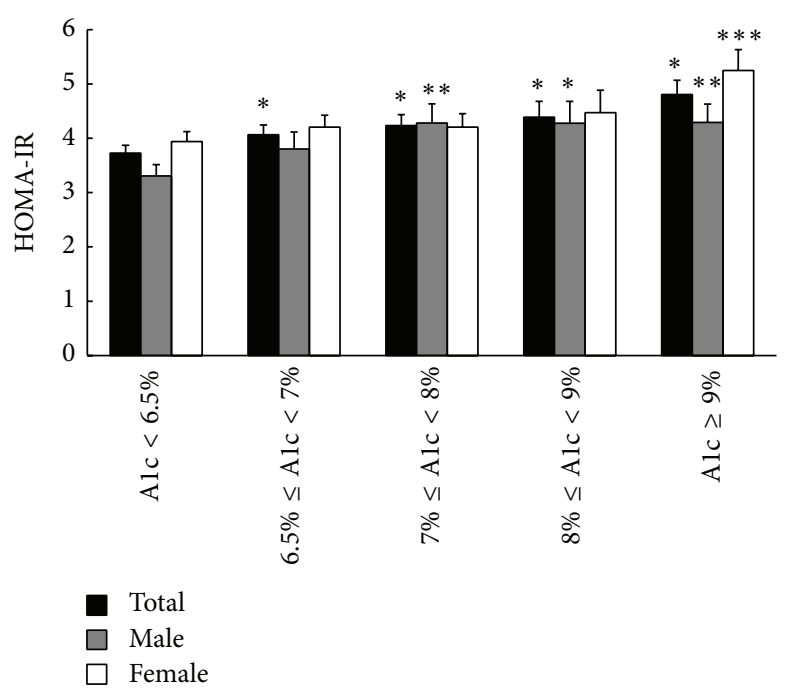

(b)

FIGURE 1: (a) $\beta$ cell function with different Alc groups (HOMA- $\beta$ ). (b) Insulin resistance with different Alc groups (HOMA-IR).

Similarly, we analyzed the association between increased Alc levels and insulin resistance using the three models (Table 2). As expected, the Alc value was significantly increased with increased insulin resistance. In Model 1, the patients with Alc values of $8 \%-9 \%$ and $\geq 9 \%$ had a significantly increased risk of insulin resistance (odds ratio $(\mathrm{OR})=$ 1.89 and 1.85, resp.). After adjusting for age, gender, BMI, and WC, group 5, with an Alc $\geq 9 \%$, also presented an increased OR (2.16). After further adjusting for systolic BP, diastolic BP, cholesterol, triglycerides, ALT, and AST, patients with an Alc value of $9 \%$ also showed an increased risk of insulin resistance $(\mathrm{OR}=2.04, P=0.014)$.

\section{Discussion}

The prevalence of diabetes has increased significantly in recent decades and is now reaching epidemic proportions in China. The most recent national survey in 2013 reported that the prevalence of diabetes and prediabetes in Chinese adults was $11.6 \%$ and $50.1 \%$, respectively [11]. The main pathophysiological defects responsible for type 2 diabetes mellitus (T2DM) are $\beta$ cell dysfunction and decreased insulin sensitivity. Pancreatic $\beta$ cell dysfunction plays a major role in determining dysglycemia from the onset of diabetes $[12,13]$. Studies from UKPDS have documented a reduction in $\beta$ cell 
TABLE 2: Logistic regression analysis of the association between different Alc groups and insulin resistance.

\begin{tabular}{|c|c|c|c|c|c|c|}
\hline \multirow{2}{*}{ Characteristics } & \multicolumn{2}{|c|}{ Model 1} & \multicolumn{2}{|c|}{ Model 2} & \multicolumn{2}{|c|}{ Model 3} \\
\hline & OR (95\% CI) & $P$ & OR (95\% CI) & $P$ & OR (95\% CI) & $P$ \\
\hline \multicolumn{7}{|l|}{ Alc groups } \\
\hline Group 1 & 1 (reference) & & 1 (reference) & & 1 (reference) & \\
\hline Group 2 & $1.30(0.82-2.05)$ & 0.264 & $0.95(0.57-1.57)$ & 0.834 & $0.91(0.54-1.53)$ & 0.708 \\
\hline Group 3 & $1.29(0.83-2.00)$ & 0.266 & $1.03(0.64-1.68)$ & 0.892 & $1.01(0.61-1.68)$ & 0.969 \\
\hline Group 4 & $1.89(1.06-3.37)$ & 0.032 & $1.66(0.86-3.21)$ & 0.134 & $1.55(0.76-3.17)$ & 0.226 \\
\hline Group 5 & $1.85(1.13-3.02)$ & 0.014 & $2.16(1.26-3.69)$ & 0.005 & $2.04(1.16-3.61)$ & 0.014 \\
\hline Female & \multicolumn{2}{|c|}{-} & $1.79(1.22-2.63)$ & 0.003 & $2.30(1.50-3.51)$ & $<0.001$ \\
\hline Age (years) & \multicolumn{2}{|c|}{-} & $0.98(0.96-0.99)$ & 0.010 & $1.00(0.98-1.02)$ & 0.882 \\
\hline BMI $\left(\mathrm{kg} / \mathrm{m}^{2}\right)$ & \multicolumn{2}{|c|}{ - } & $1.13(1.05-1.21)$ & 0.001 & $1.11(1.03-1.19)$ & 0.007 \\
\hline $\mathrm{WC}(\mathrm{cm})$ & \multicolumn{2}{|c|}{-} & $1.05(1.02-1.07)$ & $<0.001$ & $1.05(1.02-1.07)$ & 0.001 \\
\hline Systolic BP (mmHg) & \multicolumn{2}{|c|}{ - } & \multicolumn{2}{|c|}{-} & $1.00(0.98-1.02)$ & 0.772 \\
\hline Diastolic BP (mmHg) & \multicolumn{2}{|c|}{ - } & \multicolumn{2}{|c|}{-} & $1.03(1.01-1.06)$ & 0.001 \\
\hline Cholesterol (mmol/L) & \multicolumn{2}{|c|}{-} & \multicolumn{2}{|c|}{-} & $0.97(0.80-1.19)$ & 0.787 \\
\hline Triglycerides (mmol/L) & \multicolumn{2}{|c|}{-} & \multicolumn{2}{|c|}{-} & $1.17(1.02-1.33)$ & 0.021 \\
\hline AST (U/L) & \multicolumn{2}{|c|}{-} & \multicolumn{2}{|c|}{-} & $1.01(0.98-1.04)$ & 0.646 \\
\hline $\operatorname{ALT}(\mathrm{U} / \mathrm{L})$ & \multicolumn{2}{|c|}{-} & \multicolumn{2}{|c|}{-} & $1.02(0.99-1.05)$ & 0.118 \\
\hline
\end{tabular}

Model 1: unadjusted. Model 2: adjusted for age, gender, BMI, and WC. Model 3: adjusted for age, gender, BMI, WC, Systolic BP, Diastolic BP, Cholesterol, Triglycerides, AST, and ALT.

TABLE 3: Logistic regression analysis of the association between different Alc groups and impaired $\beta$ cell function.

\begin{tabular}{|c|c|c|c|c|c|c|}
\hline \multirow{2}{*}{ Characteristics } & \multicolumn{2}{|c|}{ Model 1} & \multicolumn{2}{|c|}{ Model 2} & \multicolumn{2}{|l|}{ Model 3} \\
\hline & OR (95\% CI) & $P$ & OR $(95 \% \mathrm{CI})$ & $P$ & OR (95\% CI) & $P$ \\
\hline \multicolumn{7}{|l|}{ Alc groups } \\
\hline Group 1 & 1 (reference) & & 1 (reference) & & 1 (reference) & \\
\hline Group 2 & $0.80(0.45-1.40)$ & 0.427 & $1.22(0.65-2.28)$ & 0.536 & $1.33(0.70-2.53)$ & 0.377 \\
\hline Group 3 & $1.47(0.92-2.37)$ & 0.111 & $2.47(1.42-4.27)$ & 0.001 & $2.79(1.58-4.94)$ & $<0.001$ \\
\hline Group 4 & $2.45(1.35-4.48)$ & 0.003 & $3.69(1.82-7.48)$ & $<0.001$ & $4.19(1.99-8.79)$ & $<0.001$ \\
\hline Group 5 & $15.36(9.05-26.07)$ & 0.014 & $22.08(11.86-41.12)$ & $<0.001$ & $28.51(14.53-55.95)$ & $<0.001$ \\
\hline Female & \multicolumn{2}{|c|}{-} & $0.38(0.25-0.59)$ & $<0.001$ & $0.39(0.25-0.61)$ & $<0.001$ \\
\hline Age (years) & \multicolumn{2}{|c|}{-} & $0.99(0.97-1.01)$ & 0.356 & $0.99(0.97-1.02)$ & 0.398 \\
\hline BMI $\left(\mathrm{kg} / \mathrm{m}^{2}\right)$ & \multicolumn{2}{|c|}{-} & $0.76(0.70-0.83)$ & $<0.001$ & $0.77(0.70-0.84)$ & $<0.001$ \\
\hline $\mathrm{WC}(\mathrm{cm})$ & \multicolumn{2}{|c|}{-} & $0.99(0.96-1.02)$ & 0.405 & $0.99(0.96-1.02)$ & 0.455 \\
\hline Systolic BP & \multicolumn{2}{|c|}{-} & \multicolumn{2}{|c|}{-} & $0.99(0.98-1.01)$ & 0.290 \\
\hline Diastolic BP & \multicolumn{2}{|c|}{-} & \multicolumn{2}{|c|}{ - } & $1.01(0.99-1.03)$ & 0.545 \\
\hline Cholesterol & \multicolumn{2}{|c|}{-} & \multicolumn{2}{|c|}{-} & $0.96(0.77-1.19)$ & 0.699 \\
\hline Triglycerides & \multicolumn{2}{|c|}{-} & \multicolumn{2}{|c|}{-} & $0.86(0.73-1.02)$ & 0.083 \\
\hline AST & \multicolumn{2}{|c|}{ - } & \multicolumn{2}{|c|}{-} & $1.03(0.99-1.06)$ & 0.155 \\
\hline ALT & \multicolumn{2}{|c|}{-} & \multicolumn{2}{|c|}{-} & $0.98(0.95-1.01)$ & 0.137 \\
\hline
\end{tabular}

Model 1: unadjusted. Model 2: adjusted for age, gender, BMI, and WC. Model 3: adjusted for age, gender, BMI, WC, Systolic BP, Diastolic BP, Cholesterol, Triglycerides, AST, and ALT.

function of up to $50 \%$ at the time of diagnosis, and this value gradually increases with the progression of diabetes [14]. Increasing evidence suggests that $\beta$ cell function protection should be a priority starting at the onset of diabetes.

The Alc is an integrated measure of mean glycemia over the preceding 8-12 weeks, and the ADA has recommended the Alc value as a diabetes diagnosis standard [15]. Previous studies have shown that an increase in the Alc level is usually accompanied by a decline in pancreatic $\beta$ cell function. However, whether this trend is present in newly diagnosed and drug naive diabetes patients remains unclear. Moreover, $\beta$ cell function changes at different Alc levels, and these changes in newly diagnosed T2DM patients have not been characterized. In this study, we divided the subjects into five 
groups based on their Alc levels and compared $\beta$ cell function and insulin resistance at different Alc levels. We found that individuals with an Alc of 6.5-7\% exhibited an increased HOMA- $\beta$ index compared with subjects who had an Alc < $6.5 \%$, indicating that a slight increase in the Alc level may induce increases in insulin secretion, which occur to compensate for rising insulin resistance. However, the HOMA- $\beta$ index was significantly decreased in patients with Alc levels $\geq$ $7 \%$; it was decreased by $9.3 \%$ in patients with an Alc of 7$8 \%$ and by $23.7 \%$ in patients with an Alc $8-9 \%$. As the Alc level increased to $\geq 9 \%$, a $62 \%$ reduction in $\beta$ cell function was observed, which suggests that poor glycemic control may contribute to the decrease in $\beta$ cell function. These results are consistent with a previous study [16]. Because the Alc value was significantly correlated with $\beta$ cell function in newly diagnosed and drug naive type 2 diabetes patients, our present finding could have potentially important clinical implications. Attention should be focused on the Alc value to protect $\beta$ cell function in diabetic patients. The underlying mechanisms of this process may be found in diabetic rodent studies. Sustained hyperglycaemia damages $\beta$ cell function via several mechanisms such as an increase in oxidative stress, activation of the MAPK pathway, and reduction of the pancreatic and duodenal homeobox factor-1 (PDX-1) function $[17,18]$.

In addition to hyperglycemia, the traditional risk factors of impaired $\beta$ cell function include age, obesity, hypertension, ALT, AST, and dyslipidemia. We adjusted for age, gender, BMI, systolic BP, diastolic BP, ALT, and AST, as well as WC, cholesterol, and triglyceride levels to ensure that our results were more reliable. After adjusting for the above risk factors, the Alc value was still significantly associated with impaired $\beta$ cell function in patients with Alc levels $\geq 7 \%$; no changes were observed in individuals with an Alc $<7 \%$. To date, no study has detailed insulin sensitivity in Chinese subjects with newly presented type 2 diabetes mellitus stratified by Alc levels. Our study also demonstrated that insulin resistance increased with increasing Alc levels, compared to individuals with an Alc $<6.5 \%$.

Our study also has some limitations. First, our study included only middle-aged and elderly Chinese subjects; therefore, the results might not be applicable to subjects of different ages or ethnicities. Second, we used the HOMA-IR index to evaluate insulin resistance instead of the "gold standard" (euglycemic-hyperinsulinemic clamp techniques). The HOMA-IR index is a mathematical model of the fasting state, and it represents hepatic insulin resistance only; therefore, it cannot reflect insulin resistance accurately. Third, this is only a cross-sectional study. Long-term prospective studies are needed to clarify the association between the changes in $\beta$ cell function and Alc levels during the development of diabetes.

\section{Conclusions}

Elevated Alc levels $(\geq 7 \%)$ were associated with substantial reductions in $\beta$ cell function. The Alc value could be used as simple and practical index to evaluate $\beta$ cell function and direct clinical treatment in newly diagnosed and drug naive type 2 diabetes patients.

\section{Conflict of Interests}

The authors declare that there is no conflict of interests regarding the publication of this paper.

\section{Authors' Contribution}

Xinguo Hou and Jinbo Liu contributed equally to this work.

\section{Acknowledgments}

This study was supported by grants from the National Natural Science Foundation of China (no. 81370943), Chinese Society of National Clinical Research Fund (12030420342), the National Science and Technology Support Plan (2009BAI80B04), the Ministry of Science and Technology Support Program (2013BAI09B13), the Business Plan of Jinan Students Studying Abroad (20110407), Shandong Province Outstanding Young Scientist Research Award Fund (2013BSE27128), Shandong Natural Science Foundation (no. ZR2013HQ046), Jinan Young Science and Technology Stars Project (no. 201406011), and Chinese Medical Association, clinical research and special project funds (12020170277 and 13040520437). The funders had no roles in the study design, in data collection or analysis, in the decision to publish, or in the preparation of the paper. The authors would like to acknowledge Reaction Study Group: Steering Committee: Guang Ning (Principle Investigator), National Clinical Research Center for Metabolic Diseases, Rui Jin Hospital, Shanghai Jiao Tong University School of Medicine, Shanghai, China; Yiming Mu, People's Liberation Army General Hospital, Beijing, China; Jiajun Zhao, Shandong Provincial Hospital, Jinan, China; Weiqing Wang, National Clinical Research Center for Metabolic Diseases, Rui Jin Hospital, Shanghai Jiao Tong University School of Medicine, Shanghai, China; Chao Liu, Jiangsu Province Hospital on Integration of Chinese and Western Medicine, Nanjing, China; Yufang Bi, National Clinical Research Center for Metabolic Diseases, Rui Jin Hospital, Shanghai Jiao Tong University School of Medicine, Shanghai, China; Donghui Li, Department of Gastrointestinal Medical Oncology, the University of Texas MD Anderson Cancer Center, Houston, Texas, USA; Shenghan Lai, Johns Hopkins University School of Medicine, Baltimore, Maryland, USA; Zachary T. Bloomgarden, Mount Sinai School of Medicine, New York, USA; Working Group: Weiqing Wang, Yufang $\mathrm{Bi}$, and Jieli $\mathrm{Lu}$, National Clinical Research Center for Metabolic Diseases, Rui Jin Hospital, Shanghai Jiao Tong University School of Medicine, Shanghai, China; Yiming Mu, People's Liberation Army General Hospital, Beijing, China; Jiajun Zhao, Shandong Provincial Hospital, Jinan, China; Chao Liu, Jiangsu Province Hospital on Integration of Chinese and Western Medicine, Nanjing, China; Lulu Chen, Wuhan Xiehe Hospital, Huazhong University of Science and Technology School of Medicine, Wuhan, China; Lixin Shi, Affiliated 
Hospital of Guiyang Medical College, Guiyang, China; Qiang Li, The Second Affiliated Hospital of Haerbin Medical University, Haerbin, China; Tao Yang, The First Affiliated Hospital with Nanjing Medical University, Jiangsu Province Hospital, Nanjing, China; Li Yan, Sun Yat-sen Memorial Hospital, Sun Yat-sen University, Guangzhou, China; Qin Wan, The Affiliated Hospital of Luzhou Medical College, Luzhou, China; Shengli Wu, Xinjiang Kelamayi Peoples Hospital, Kelamayi, China; Guixia Wang, The First Hospital of Jilin University, Changchun, China; Zuojie Luo, The First Affiliated Hospital of Guangxi Medical University, Nanning, China; Xulei Tang, The First Hospital of Lanzhou University, Lanzhou, China; Gang Chen, Fujian Provincial Hospital, Fujian Medical University, Fuzhou, China; Yanan Huo, Jiangxi People's Hospital, Nanchang, China; Zhengnan Gao, Dalian Municipal Central Hospital, Dalian, China; Qing Su, Xinhua Hospital, Shanghai Jiao Tong University School of Medicine, Shanghai, China; Zhen Ye, Zhejiang Center for Disease Control, China; Youmin Wang, The First Affiliated Hospital of Anhui Medical University, Hefei, China; Guijun Qin, The First Affiliated Hospital of Zhengzhou University, Zhengzhou, China; Huacong Deng, The First Affiliated Hospital of Chongqing Medical University, Chongqing, China; Xuefeng Yu, Wuhan Tongji Hospital, Huazhong University of Science and Technology School of Medicine, Wuhan, China; Feixia Shen, The First Affiliated Hospital of Wenzhou Medical University, The First Provincial Wenzhou Hospital of Zhejiang, Wenzhou, China; and Li Chen, Qilu Hospital of Shandong University, Jinan, China.

\section{References}

[1] R. A. DeFronzo, "Pathogenesis of type 2 diabetes mellitus," Medical Clinics of North America, vol. 88, no. 4, pp. 787-835, 2004.

[2] C. Weyer, C. Bogardus, D. M. Mott, and R. E. Pratley, “The natural history of insulin secretory dysfunction and insulin resistance in the pathogenesis of type 2 diabetes mellitus," Journal of Clinical Investigation, vol. 104, no. 6, pp. 787-794, 1999.

[3] V. Poitout and R. P. Robertson, "Minireview:secondary beta-cell failure in type 2 diabetes-a convergence of glucotoxicity and lipotoxicity," Endocrinology, vol. 143, no. 2, pp. 339-342, 2002.

[4] D. M. Nathan, J. Kuenen, R. Borg, H. Zheng, D. Schoenfeld, and R. J. Heine, "Translating the A1C assay into estimated average glucose values," Diabetes Care, vol. 31, no. 8, pp. 1473-1478, 2008.

[5] G. Ning, N. Guang, J. Shanghai et al., "Risk Evaluation of cAncers in Chinese diabeTic Individuals: a lONgitudinal (REACTION) study," Journal of Diabetes, vol. 4, no. 2, pp. 172173, 2012.

[6] Y. Bi, J. Lu, W. Wang et al., "Cohort profile: risk evaluation of cancers in Chinese diabetic individuals: a longitudinal (REACTION) study," Journal of Diabetes, vol. 6, no. 2, pp. 147157, 2014.

[7] J. Lu, Y. Bi, T. Wang et al., "The relationship between insulinsensitive obesity and cardiovascular diseases in a Chinese population: results of the REACTION study," International Journal of Cardiology, vol. 172, no. 2, pp. 388-394, 2014.

[8] G. Ning and Z. Bloomgarden, "Diabetes and cancer: findings from the REACTION study," Journal of Diabetes, vol. 7, no. 2, pp. 143-144, 2015.
[9] D. R. Matthews, J. P. Hosker, A. S. Rudenski, B. A. Naylor, D. F. Treacher, and R. C. Turner, "Homeostasis model assessment: insulin resistance and beta-cell function from fasting plasma glucose and insulin concentrations in man," Diabetologia, vol. 28, no. 7, pp. 412-419, 1985.

[10] K. G. M. M. Alberti and P. Z. Zimmet, "Definition, diagnosis and classification of diabetes mellitus and its complications. Part 1: diagnosis and classification of diabetes mellitus. Provisional report of a WHO consultation," Diabetic Medicine, vol. 15, no. 7, pp. 539-553, 1998.

[11] Y. Xu, L. Wang, J. He et al., "Prevalence and control of diabetes in Chinese adults," The Journal of the American Medical Association, vol. 310, no. 9, pp. 948-959, 2013.

[12] R. A. DeFronzo and M. A. Abdul-Ghani, "Preservation of $\beta$ cell function: the key to diabetes prevention," Journal of Clinical Endocrinology and Metabolism, vol. 96, no. 8, pp. 2354-2366, 2011.

[13] P. Marchetti, R. Lupi, S. del Guerra et al., "Goals of treatment for type 2 diabetes: beta-cell preservation for glycemic control," Diabetes Care, vol. 32, supplement 2, pp. S178-S183, 2009.

[14] 'U.K. prospective diabetes study 16. Overview of 6 years' therapy of type II diabetes: a progressive disease. U.K. Prospective Diabetes Study Group," Diabetes, vol. 44, no. 11, pp. 1249-1258, 1995.

[15] R. W. Grant and M. S. Kirkman, "Trends in the evidence level for the American Diabetes Association's 'Standards of Medical Care in Diabetes' from 2005 to 2014," Diabetes Care, vol. 38, no. 1, pp. 6-8, 2005.

[16] F. S. Fang, X. L. Cheng, Y. P. Gong et al., "Association between glycemic indices and beta cell function in patients with newly diagnosed type 2 diabetes," Current Medical Research and Opinion, vol. 30, no. 8, pp. 1437-1440, 2014.

[17] E. Zini, M. Osto, M. Franchini et al., "Hyperglycaemia but not hyperlipidaemia causes beta cell dysfunction and beta cell loss in the domestic cat," Diabetologia, vol. 52, no. 2, pp. 336-346, 2009.

[18] H. Kaneto, Y. Nakatani, D. Kawamori, T. Miyatsuka, and T. A. Matsuoka, "Involvement of oxidative stress and the JNK pathway in glucose toxicity," The Review of Diabetic Studies, vol. 1, no. 4, pp. 165-174, 2004. 


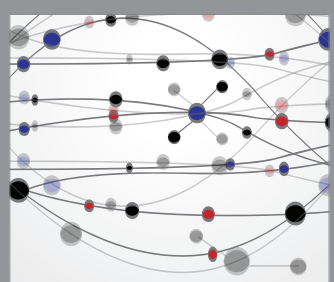

The Scientific World Journal
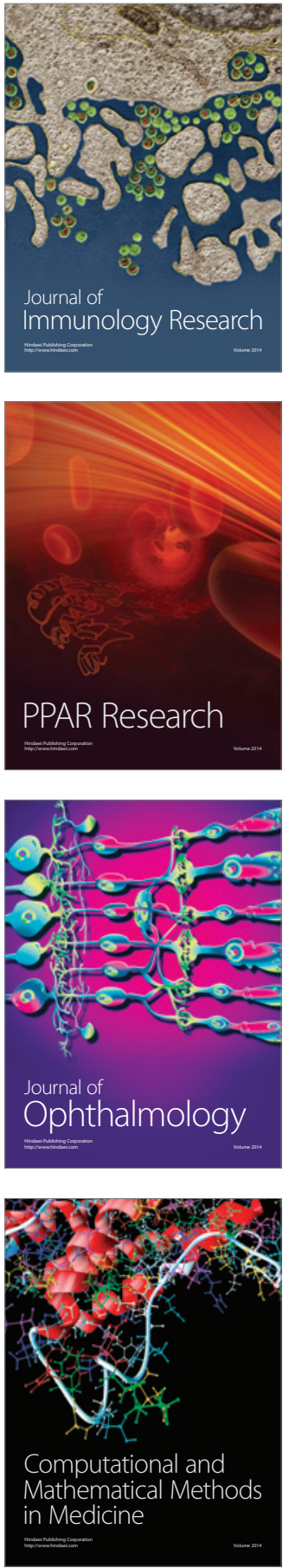

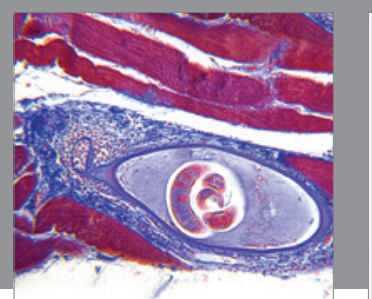

Gastroenterology Research and Practice

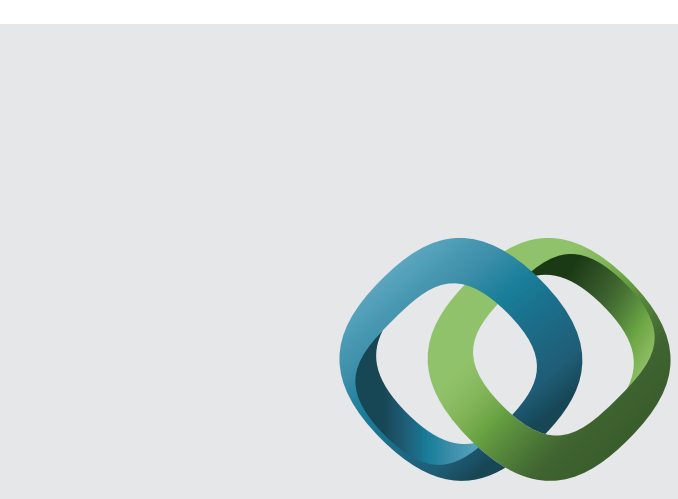

\section{Hindawi}

Submit your manuscripts at

http://www.hindawi.com
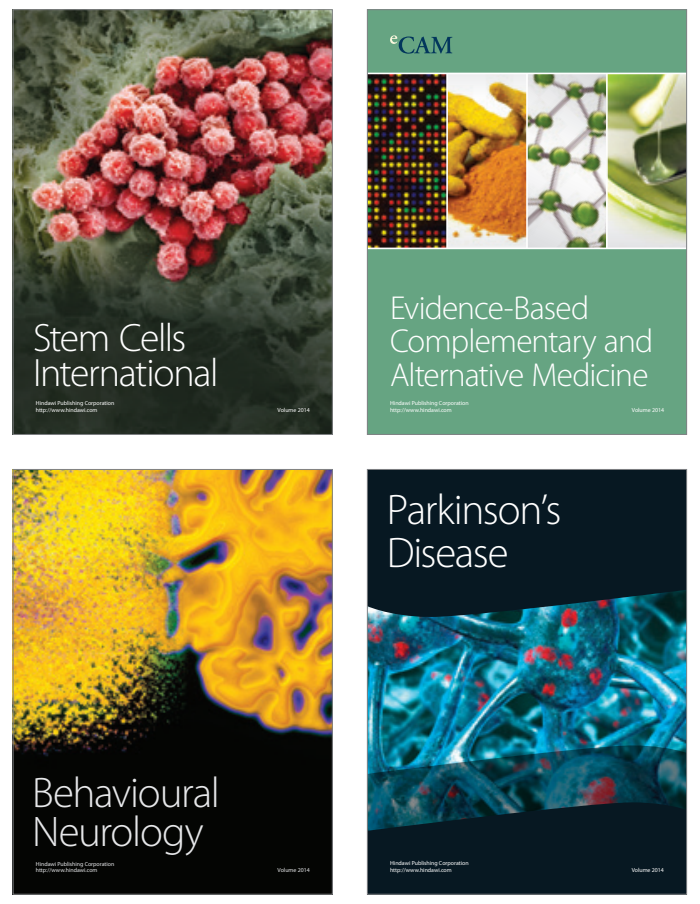
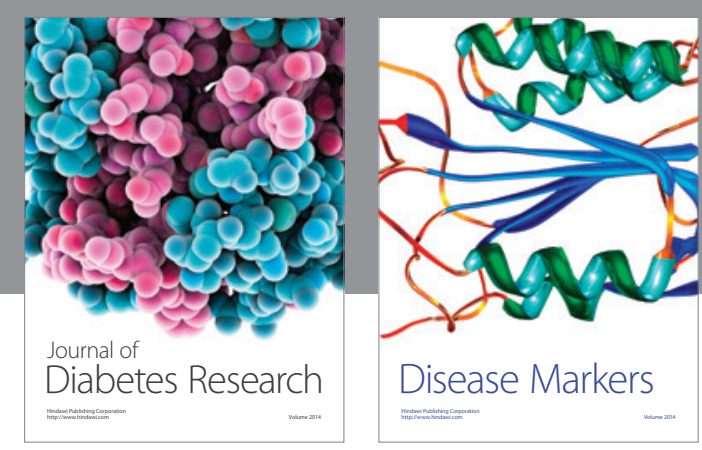

Disease Markers
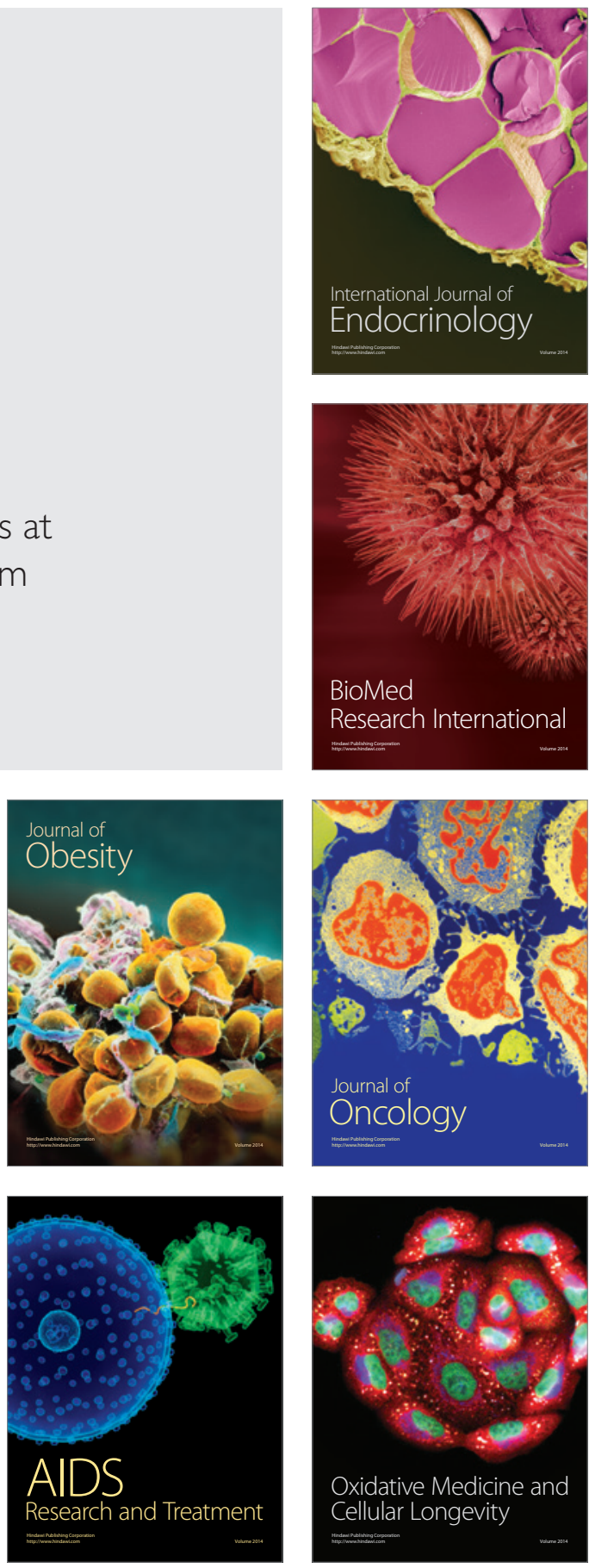